\title{
Loss of ARID1A/BAF250a-expression in endometriosis: a biomarker for risk of carcinogenic transformation?
}

\author{
Eleftherios P Samartzis ${ }^{1}$, Nicolas Samartzis ${ }^{1}$, Aurelia Noske ${ }^{2}$, André Fedier ${ }^{1}$, \\ Rosmarie Caduff ${ }^{2}$, Konstantin J Dedes ${ }^{1}$, Daniel Fink ${ }^{1}$ and Patrick Imesch ${ }^{1}$ \\ ${ }^{1}$ Department of Gynecology, University Hospital Zurich, Zurich, Switzerland and ${ }^{2}$ Department of Pathology, \\ University Hospital Zurich, Zurich, Switzerland
}

\begin{abstract}
Mutations of the tumor-suppressor gene ARID1A result in the loss of protein expression of the BRG-associated factor 250a (BAF250a), a large subunit of transcription-regulating Human SWI/SNF complexes, which have an important role in the control of cell proliferation and tumor suppression. ARID1A mutations are particularly frequent in endometriosis-associated ovarian clear cell and endometrioid carcinomas, and were recently described as a possible key mechanism and early step in the transformation of endometriosis into cancer. Here, we examined the immunohistochemical expression pattern of BAF250a in a tissue microarray including 74 endometriosis and 30 endometrium samples. Ovarian cancer samples $(n=136)$ served as a control. Epithelial BAF250a expression was assessable in 90/104 (87\%) and stromal BAF250a expression in 95/104 (91\%) of the endometriosis, and endometrium cases due to lack of adequate tissue in some spots. Complete lack of BAF250a expression was observed in three endometriomas $(n=3 / 20,15 \%)$ and one deep-infiltrating endometriosis sample $(n=1 / 22,5 \%)$, but in none of the peritoneal endometriosis $(n=0 / 16)$ and eutopic endometrium samples $(n=0 / 30)$. A comparison of the mean immunoreactivity scores revealed a significantly lower expression rate of BAF250a in endometriomas compared with normal endometrium $(P<0.0005)$, as well as peritoneal $(P=0.003)$ and deep-infiltrating endometriosis $(P=0.02)$. Our data demonstrates that a complete loss of BAF250a expression is observable in some endometriotic lesions, especially in endometriomas. In addition, we report that a partial loss of BAF250a expression is occurring in the form of cell clusters indicating a clonal loss of BAF250a expression in these cells. The loss of expression of the tumor-suppressor protein BAF250a in some endometriomas possibly indicates a risk of malignant transformation in these cases, which could be of importance in the determination of individual treatment strategies. However, its role and value as a prognostic parameter in endometriosis needs to be further studied.
\end{abstract}

Modern Pathology (2012) 25, 885-892; doi:10.1038/modpathol.2011.217; published online 3 February 2012

Keywords: ARID1A; BAF250a; endometriosis; ovarian carcinomas

Endometriosis is an estrogen-dependent inflammatory disease affecting $5-10 \%$ of women of reproductive age. ${ }^{1,2}$ It is defined by the presence of ectopic endometrium-like tissue, and is clinically characterized by chronic pelvic pain, dysmenorrhea, dyspareunia, and infertility. ${ }^{1,2}$ Although endometriosis is a benign disease, a clinical association with ovarian cancer, in particular ovarian clear cell carcinomas

Correspondence: Dr P Imesch, MD, University Hospital Zurich, Department of Gynecology, Frauenklinikstrasse 10, CH-8091 Zurich, Switzerland.

E-mail: patrick.imesch@usz.ch

Received 23 June 2011; revised 1 December 2011; accepted 9

December 2011; published online 3 February 2012 and endometrioid ovarian carcinomas, has repeatedly been observed in the past years. ${ }^{3-5}$ There is growing evidence, that endometriosis is linked to ovarian clear cell carcinomas and endometrioid ovarian carcinomas through distinct pathomechanisms. ${ }^{5,6}$ One of them is possibly linked to mutations that result in the subsequent loss of the tumorsuppressor gene AT rich interactive domain 1A (ARID1A). Using whole-exome sequencing, recurrent mutations in ARID1A were recently reported in ovarian clear cell carcinomas and endometrioid ovarian carcinomas by two independent groups., ${ }^{7,8}$ Wiegand et $a 7^{7}$ found mutations in ARID1A in $46 \%$ of 119 ovarian clear cell carcinomas and $30 \%$ of 33 endometrioid ovarian carcinomas but in none of 76 
high-grade serous carcinomas. Likewise, Jones et al ${ }^{8}$ observed ARID1A-mutations in $57 \%$ of 42 ovarian clear cell carcinomas. Using immunohistochemistry it was demonstrated that ARID1A mutations result in the loss of the protein BRG-associated factor 250a (BAF250a), which is a large subunit of transcriptionregulating Human SWI/SNF complexes and has an important role in the control of cell proliferation and tumor suppression. ${ }^{7,9,10}$ Recently, Guan et al ${ }^{11}$ found somatic ARID1A mutations in 10/25 uterine endometrioid carcinomas, and could also confirm a strong correlation to loss of BAF250a expression by immunohistochemistry.

Further evidence for the importance of mutations in ARID1A in the pathogenesis of endometriosisassociated carcinomas could be seen in two cases, where loss of BAF250a protein expression was observable simultaneously in ARID1A mutated tumors and contiguous atypical endometriosis but not in distant endometriosis. ${ }^{7}$ Loss of BAF250a expression could therefore represent a key mechanism and early step in the transformation of endometriosis into cancer. ${ }^{7,8}$ The aim of this study is to examine if loss of BAF250a expression is present in benign endometriotic tissue. Therefore, we examined a large cohort of endometriotic lesions as well as a control cohort of ovarian cancer by immunohistochemistry. As loss of expression of this tumor-suppressor gene is regarded to be an early and crucial mechanism in endometriosis-associated ovarian carcinomas $^{7,11}$ it could be an important indicator of a risk for carcinogenic transformation.

\section{Materials and methods}

\section{Patients and Tissue Specimens}

The study was approved by the local ethical committee (StV 27-2009 and KEK-ZH-2010-0174/0). ARID1A (BAF250a) expression was examined by immunohistochemistry in two tissue microarrays, one consisting of study cases (ectopic endometriotic tissue and eutopic endometrium) and one of control cases (different ovarian carcinoma subtypes). Clinical and pathological characteristics for the study and the control tissue microarray were collected from the clinical database of the gynecological department and the pathology records of the University Hospital of Zurich.

The study cases included 74 samples of 71 patients with ectopic typical endometriotic tissue (19 peritoneal, 28 deep infiltrating and 27 ovarian) undergoing laparoscopic endometriosis treatment as well as 30 samples of healthy eutopic endometrium from patients undergoing hysterectomies for predominantly benign conditions such as uterine myomas or prolapsed uterus. All tissue samples originated from premenopausal patients. The diagnosis of endometriosis was initially done at the Institute of Pathology of the University Hospital Zurich between
2000 and 2010, and all included cases were reviewed for this study by a gynecologic pathologist.

The control cases consisted of 136 primary epithelial ovarian carcinomas (66 serous, 24 clear cell, 31 endometrioid and 15 mucinous) as well as 3 samples of the fallopian tube. These patients were surgically treated at the University Hospital of Zurich (Switzerland) between 1995 and 2005. The stage of tumors was assessed according to the International Federation of Gynecology and Obstetrics (FIGO) staging system. The tumor grade and histological subtype were defined according to the WHO classification 2003.

\section{Tissue Microarray}

The tissue microarrays were constructed using a semiautomatic tissue arrayer (Beecher Instruments, Woodland, TX, USA) as previously described. ${ }^{12}$ Tissue samples were fixed in $4 \%$ neutral buffered formaldehyde and were embedded in paraffin. Routine hematoxylin/eosin-stained sections were performed for histopathological evaluation. Areas involving endometriotic or tumor tissue were marked respectively. Samples were further selected under the term of tissue availability. Two cylindrical tissue cores of $0.6 \mathrm{~mm}$ diameter were punched out from each case of the corresponding paraffin-embedded blocks and inserted into the recipient blocks.

The study tissue microarray block was freshly cut in two-and-a-half micrometer sections and mounted on superfrost slides (Menzel Gläser, Braunschweig, Germany). After antigen retrieval with CC1m- heatinduced epitope retrieval, the slides were incubated with a monoclonal mouse ARID1A antibody (ABGENT, code: AT1188a), as previously validated, ${ }^{7,11,13}$ which was diluted 1:200 in Ventana dilution buffer. After incubation for $1 \mathrm{~h}$ at room temperature, the staining was further conducted with the Ventana Benchmark automated system (Ventana Medical Systems, Tuscon, AZ, USA). Slides were counterstained with haematoxylin, dehydrated and mounted. The expression analysis was performed by two authors (AN, NS) and was evaluated according to the percentage of positive cells and the staining intensity. Nuclear immunoreactivity was considered as a positive expression. Endothelial cells served as positive internal controls. All BAF250a negative endometriotic cases were confirmed on whole tissue sections. Negative controls were performed by replacement of the primary antibody by non-reactive rabbit IgG (DAKO). Identical procedure was applied for the control tissue microarray block.

\section{Scoring and Statistical Analysis}

The percentage of positive cells was scored as: 0 $(0 \%) ;+(\leq 10 \%) ;++(11-50 \%) ;+++(51-80 \%) ;$ and $++++(>80 \%)$. The staining intensity was defined as: $0^{*}$ (negative), $1^{*}$ (weak), $2^{*}$ (moderate) 
and $3^{*}$ (strong). For the immunoreactive score,${ }^{14}$ the percentage of positive cells and staining intensity was multiplicated, resulting in a value between 0 and 12 .

SPSS software (Version 19.0, SPSS, Chicago, IL, USA) was used for the statistical evaluation. Generally, $P$-values $<0.05$ were considered as significant. Values are indicated as percentage and absolute numbers, as mean and standard deviation ( \pm s.d.) or as median and interquartile range (IQR), respectively. BAF250a expression rates were compared by a $\chi^{2}$ test for trends. Immunoreactivity scores were compared by a Mann-Whitney test or a Kruskal-Wallis test where appropriate. Scoring and statistical methods were identical in the study and control cases.

The statistical significance of the association between BAF250a expression and clinico-pathological parameters was assessed by a $\chi^{2}$ test for trends, a Fisher's exact test or a Spearman's $\rho$ test (bivariat correlation analysis) where appropriate.

\section{Results}

Patients with ovarian endometriosis had a mean age ( \pm s.d., range) of $35( \pm 7,19-48)$ years, with peritoneal endometriosis 31 ( $\pm 4,25-38$ ) years and with deep infiltrating endometriosis 35 ( $\pm 5,25-42)$ years. The average age of the patients with normal uterine endometrium samples was 40 ( $\pm 3,29-44)$ years. Clinical endometriosis severity score following the American Society for Reproductive Medicine (ASRM) was known for 58/71 (82\%) endometriosis patients, with following distribution: ASRM-I, 4/71 (6\%); ASRM-II, 7/71 (10\%); ASRM-III, 15/71 (21\%); and ASRM-IV, 32/71 (45\%). Three patients in the study group had a diagnosis of malignant disease. Two of them were cervical carcinomas and were in the eutopic endometrium group and one was a cervical carcinoma with simultaneous pulmonary adenocarcinoma and was in the deep-infiltrating endometriosis group. In particular, no ovarian carcinomas were observed in the study group prior or during the study time. However, all endometriosis cases were from relatively recent time and hence no longer clinical follow-up was available.

In the control group with ovarian tumors, the mean patient age at the time of surgery was $59( \pm 14)$ years, ranging from 20 to 87 . The median follow-up time of the surviving patients was 59 months. FIGO stages were known in 131 cases as follows: I, 33/131 (25\%); II, 10/131 (8\%); III, 50/131 (38\%); and IV, 38/ 131 (29\%). Tumor grades were: G1, 22/136 (16\%); G2, 58/136 (43\%); and G3, 56/136 (41\%). Residual disease status was reported in 90 cases, thereof $<1 \mathrm{~cm}$ for $26(29 \%)$ cases, $1-2 \mathrm{~cm}$ for $27(30 \%)$ and $>2 \mathrm{~cm}$ for 37 (41\%). Data on adjuvant chemotherapy was known for all patients and in the majority a platinum-based combination therapy was administered.
Table 1 Epithelial and stromal BAF250a (ARID1A) expression

\begin{tabular}{llllll}
\hline Tissue type & \multicolumn{4}{c}{ BAF250a epithelial } & Total \\
\cline { 2 - 6 } & 0 & + & ++ & +++ & ++++ \\
\end{tabular}

Endometrium $(\mathrm{P}=0.001)^{a}$
BAF250a stromal

$\begin{array}{lllllrr}0 & 0 & 1 & 0 & 0 & 1 & 2 \\ + & 0 & 0 & 0 & 1 & 0 & 1 \\ ++ & 0 & 0 & 0 & 1 & 8 & 9 \\ +++ & 0 & 0 & 0 & 0 & 5 & 5 \\ ++++ & 0 & 0 & 0 & 0 & 13 & 13 \\ \text { Total } & 0 & 1 & 0 & 2 & 27 & 30\end{array}$

Endometriosis (ovarian) $(\mathrm{P}=0.002)^{a}$

BAF250a stromal

$\begin{array}{lllllrr}0 & 3 & 0 & 0 & 0 & 0 & 3 \\ + & 0 & 0 & 0 & 1 & 0 & 1 \\ ++ & 0 & 0 & 1 & 0 & 2 & 3 \\ +++ & 0 & 0 & 1 & 2 & 5 & 8 \\ ++++ & 0 & 0 & 0 & 0 & 5 & 5 \\ \text { Total } & 3 & 0 & 2 & 3 & 12 & 20\end{array}$

Endometriosis (peritoneal) $(\mathrm{P}=0.003)^{a}$

BAF250a stromal

$\begin{array}{lllllrr}0 & 0 & 0 & 0 & 1 & 1 & 2 \\ + & 0 & 0 & 1 & 0 & 0 & 1 \\ ++ & 0 & 0 & 0 & 0 & 4 & 4 \\ +++ & 0 & 0 & 0 & 0 & 3 & 3 \\ ++++ & 0 & 0 & 0 & 0 & 6 & 6 \\ \text { Total } & 0 & 0 & 1 & 1 & 14 & 16\end{array}$

Endometriosis (deep) $(\mathrm{P}=0.001)^{a}$

BAF250a stromal

$\begin{array}{lrlllrr}0 & 1 & 0 & 0 & 0 & 0 & 1 \\ + & 0 & 0 & 0 & 0 & 0 & 0 \\ ++ & 0 & 0 & 0 & 0 & 7 & 7 \\ +++ & 0 & 0 & 0 & 0 & 9 & 9 \\ ++++ & 0 & 0 & 1 & 0 & 4 & 5 \\ \text { Total } & 1 & 0 & 1 & 0 & 20 & 22\end{array}$

Total $(\mathrm{P}=0.001)^{a}$

BAF250a stromal

$\begin{array}{lrrrrrr}0 & 4 & 1 & 0 & 1 & 2 & 8 \\ + & 0 & 0 & 1 & 2 & 0 & 3 \\ ++ & 0 & 0 & 1 & 1 & 21 & 23 \\ +++ & 0 & 0 & 1 & 2 & 22 & 25 \\ ++++ & 0 & 0 & 1 & 0 & 28 & 29 \\ \text { Total } & 4 & 1 & 4 & 6 & 73 & 88\end{array}$

$0,0 \%$ of the cells stained positive; + , up to $10 \%$ of the cells;,$++ 11-$ $50 \%$ of the cells;,$+++ 51-80 \% ;++++,>80 \%$ of the cells.

Representation of only the cases that were simultaneously evaluable for epithelial and stromal BAF250a (ARID1A) expression. Cases that could only be evaluated for either epithelial or stromal expression due to lack of adequate tissue in the sample are not included in this table. ${ }^{\mathrm{a}} \chi^{2}$ test.

Immunohistochemical staining of BAF250a (epithelial and/or stromal) was assessable in 102/ $104(98 \%)$ of the study cases (endometriosis and endometrium) due to the lack of adequate tissue in some tissue microarray spots. Epithelial BAF250a expression was assessable in 90/104 (87\%) of the cases (endometriomas 21/27 (78\%), peritoneal endometriosis 17/19 (90\%), deep-infiltrating endometriosis 22/28 (79\%) and eutopic endometrium 30/30 $(100 \%))$. Stromal BAF250a expression was assessable 
in 95/104 (91\%) of the cases (endometriomas 24/27 (89\%), peritoneal endometriosis 16/19 (84\%), deepinfiltrating endometriosis 25/28 (89\%) and eutopic endometrium 30/30 (100\%)).

Complete lack of epithelial as well as stromal BAF250a expression was observed in three endometriomas $(n=3 / 20,15 \%)$ and one deep-infiltrating endometriosis sample $(n=1 / 22,5 \%)$, and was confirmed by whole tissue sections. There were no negative cases in peritoneal endometriosis and in eutopic endometrium.

Epithelial and stromal BAF250a expression correlated strongly in all endometriosis types as well as in eutopic endometrium. In the cases with heterogeneous BAF250a expression, loss of BAF250a expression principally occurred in large groups of adjacent endometriotic cells, whereas other cell groups in the same sample clearly showed homogeneous positivity for BAF250a. This phenomenon is also reported as 'cell clusters' or 'clonal loss' in the discussion part of this work. The expression pattern of BAF250a in epithelial and stromal endometriosis and uterine endometrium cells is reported in detail in Table 1 . Representative images are shown in Figures 1a-d.

The median immunoreactivity score for epithelial and stromal BAF250a expression in endometriotic lesions was 4 and 3 , respectively. The median immunoreactivity score for epithelial BAF250a expression in ectopic endometriosis samples and in eutopic endometrium were 4 (IQR, 2-6) and 6 (IQR, 4-8), respectively $(P=0.008)$. Considering each endometriosis subgroup, median immunoreactivity score of epithelial BAF250a expression was 2 (IQR, 2-4) in ovarian endometriosis, 6 (IQR, 3.5-8) in peritoneal endometriosis and 4 (IQR, 3-6) in deep-infiltrating endometriosis. Expression rates of epithelial BAF250a were significantly lower in ovarian endometriosis compared with eutopic endometrium $(P<0.0005)$, to peritoneal endometriosis $(P=0.003)$ and to deepinfiltrating endometriosis $(P=0.02)$, as represented in Figure 2.

Immunohistochemical analysis was successful in $129 / 136$ ovarian carcinomas owing to the lack of
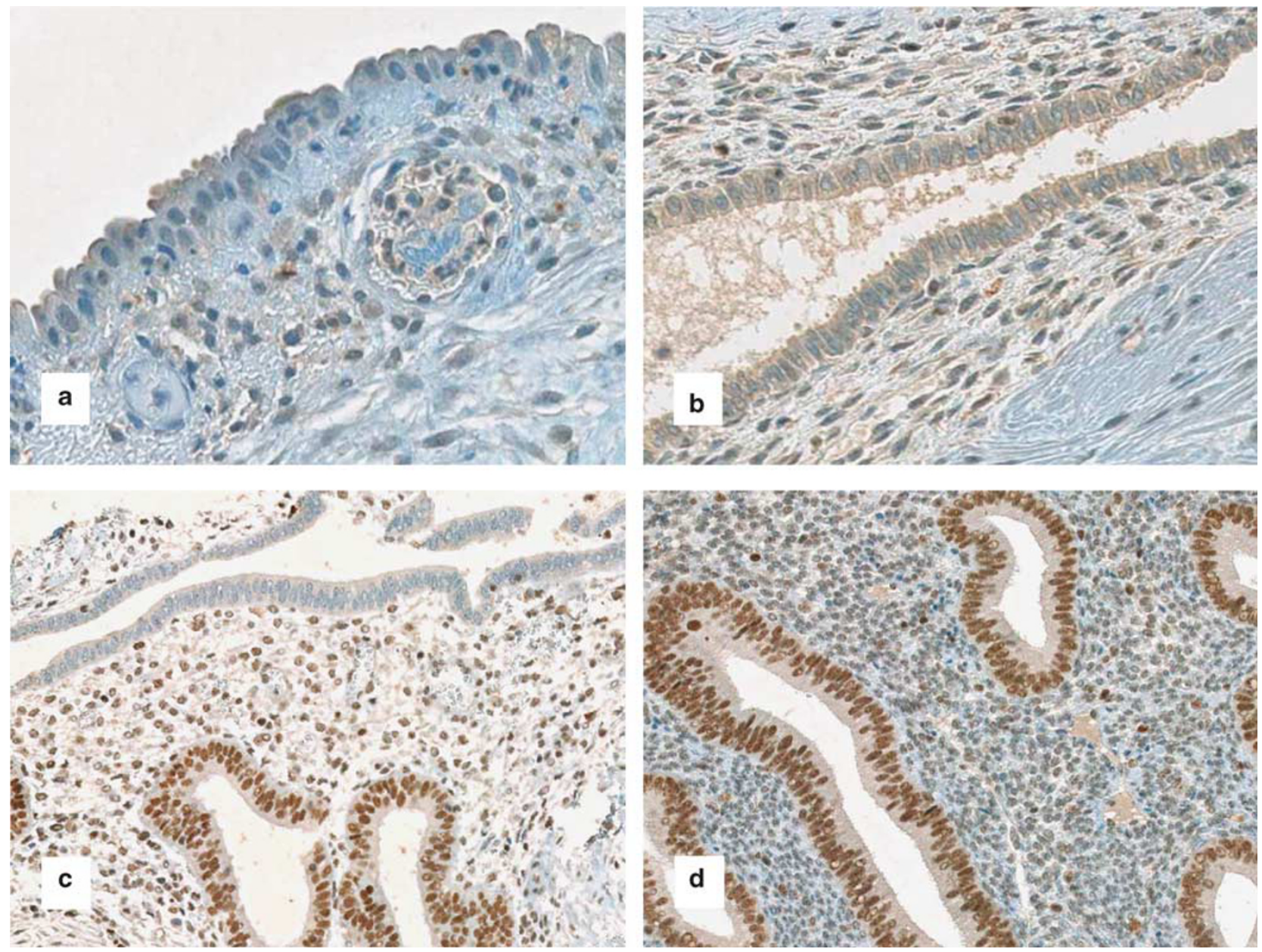

Figure 1 BAF250a expression in endometriosis. Immunohistochemical staining of ARID1A/BAF250a in endometriosis compared with normal endometrium. (a and $\mathbf{b})$ : Examples of complete loss of BAF250a expression in epithelium and adjacent stroma of ovarian endometrioma $(\mathbf{a}, \times 200)$ as well as deep-infiltrating endometriosis $(\mathbf{b}, \times 200)$. (c) Cluster-like epithelial deficiency of BAF250a expression in an ovarian endometrioma $(\times 200)$. (d) Strong nuclear BAF250a expression in normal endometrium $(\times 200)$. 


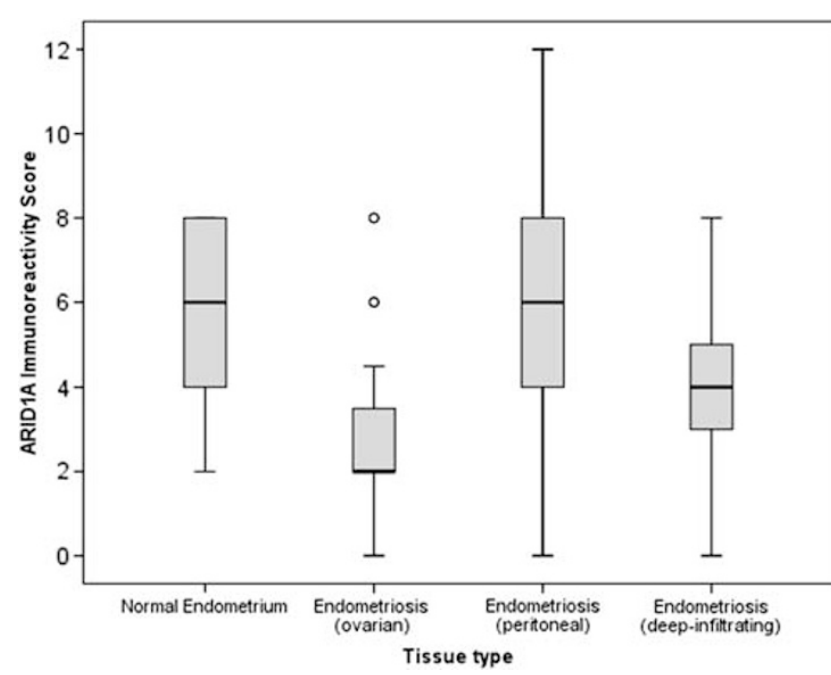

Figure 2 Immunoreactivity score in endometriosis and endometrium. The immunoreactivity score ${ }^{14}$ for BAF250a was significantly lower in ovarian endometriosis compared with eutopic endometrium $(P<0.0005)$, to peritoneal endometriosis $(P=0.003)$ and to deep-infiltrating endometriosis $(P=0.02)$, as represented in this chart diagram (calculated with a Kruskal-Wallis test).

tumor tissue in some tissue microarray spots. We observed a completely negative expression of BAF250a in $29(23 \%)$ of the 129 carcinomas. Regarding the different carcinoma subtypes $22 \%(n=5 / 23)$ of the ovarian clear cell carcinomas, $46 \%(n=13 / 28)$ of the endometrioid ovarian carcinomas, $27 \%(n=4 / 15)$ of the mucinous and $11 \%(n=7 / 63)$ of the serous ovarian carcinomas stained completely BAF250a negative. Representative images are shown in Figures 3a-d. Epithelium of the fallopian tube stained BAF250a positive in all evaluable cases $(n=3)$. We found that negative BAF250a expression was significantly associated with clinico-pathological factors like FIGO stage, tumor- and nodal stage, endometrioid histological subtype, intraoperative residual disease and patient age but not with tumor differentiation (grade) as indicated in Table 2.

\section{Discussion}

Transformation of endometriosis into cancer is a rare event. ${ }^{15}$ The pathogenesis, however, is still not well understood ${ }^{5,16}$ Historically, Sampson ${ }^{17}$ reported the first case of malignant transformation of endometriosis into an ovarian carcinoma. In the past years there is growing evidence for the relevance of a pathogenic link between these two diseases. ${ }^{6}$

Several studies indicated that in women with ovarian endometriosis there is an increased risk for the development of epithelial ovarian cancer, especially the histological subtypes of ovarian clear cell carcinomas and endometrioid ovarian carcinomas. ${ }^{4,16,18-22}$ Brinton et $a l^{18}$ reported a 4.2-fold risk for ovarian cancer in cases of long-standing history of ovarian endometriosis in a cohort of 20686 women hospitalized with endometriosis. Melin et al ${ }^{19}$ confirmed an increased risk for ovarian cancer in a cohort of 64492 women in cases with long-standing endometriosis. Kobayashi et $a l^{4}$ reported 46 cases of ovarian carcinomas in a large prospective population-based Japanese study with an ovarian endometriosis cohort of 6398 women and a follow-up of up to 17 years. It was shown that women with ovarian endometriomas in this cohort had a ninefold increased risk to develop ovarian cancer., ${ }^{3,4}$ As shown on the other hand by Vercellini et $a l^{20}$ in 556 patients that underwent surgery for ovarian cancers, the frequency of simultaneous endometriosis was also significantly higher with $26.3,21.1$ and $22.2 \%$ in cases with endometrioid ovarian carcinomas, ovarian clear cell carcinomas or mixed subtypes, respectively, compared with $3.6-5.6 \%$ in serous, mucinous and miscellaneous ovarian cancers. In another study with 127 patients with primary ovarian carcinomas endometriosis rates of $70 \%$ were reported for ovarian clear cell carcinomas (30/43) and $43 \%$ for endometrioid ovarian carcinomas (3/7), whereas only $7 \%$ of the serous carcinomas (4/60) and none of the mucinous carcinomas (0/17) showed simultaneous endometriosis. Moreover, the authors described 29 cases of atypical endometriosis in this cohort and demonstrated evidence for the transition from atypical endometriosis to carcinoma in 23 cases. ${ }^{21}$ Another study reported an endometriosis rate of $37 \%$ in a large series of 221 patients with endometrioid ovarian carcinomas. ${ }^{23}$

Epidemiological data indicates an early onset of endometriosis-associated ovarian carcinomas such as ovarian clear cell carcinomas and endometrioid ovarian carcinomas. ${ }^{3,4,24}$ In western countries ovarian clear cell carcinomas account for $12 \%$ of epithelial ovarian carcinomas and endometrioid ovarian carcinomas for $11 \%$, whereas serous carcinomas are the most common subtype with $68 \%{ }^{25}$ In Asian countries as in Japan, the percentage of ovarian clear cell carcinomas is even higher with more than $20 \%,{ }^{26}$ which is remarkable as studies report a higher prevalence of endometriosis in Asian women. ${ }^{27}$ Ovarian clear cell carcinomas are relatively resistant to conventional platinum- or taxane-based therapy and are associated with a poor prognosis especially in more advanced stages. ${ }^{3,26,28}$ Identification of biomarkers for an increased risk of malignant transformation in endometriosis could improve early detection of endometriosis-associated epithelial ovarian cancers. Lack of BAF250a, as a potential early event in the carcinogenesis, could possibly serve as screening for endometriotic lesions at high-risk for the development of ovarian clear cell carcinomas or endometrioid ovarian carcinomas. ${ }^{29}$

We used immunohistochemical staining of BAF250a $(A R I D 1 A)$ as a surrogate marker for ARID1A mutations as previously validated,, ${ }^{7,11,13}$ as the small endometriotic samples obtained after laparoscopic biopsies would not yield a sufficient amount of DNA for screening ARID1A mutations after microdissection 

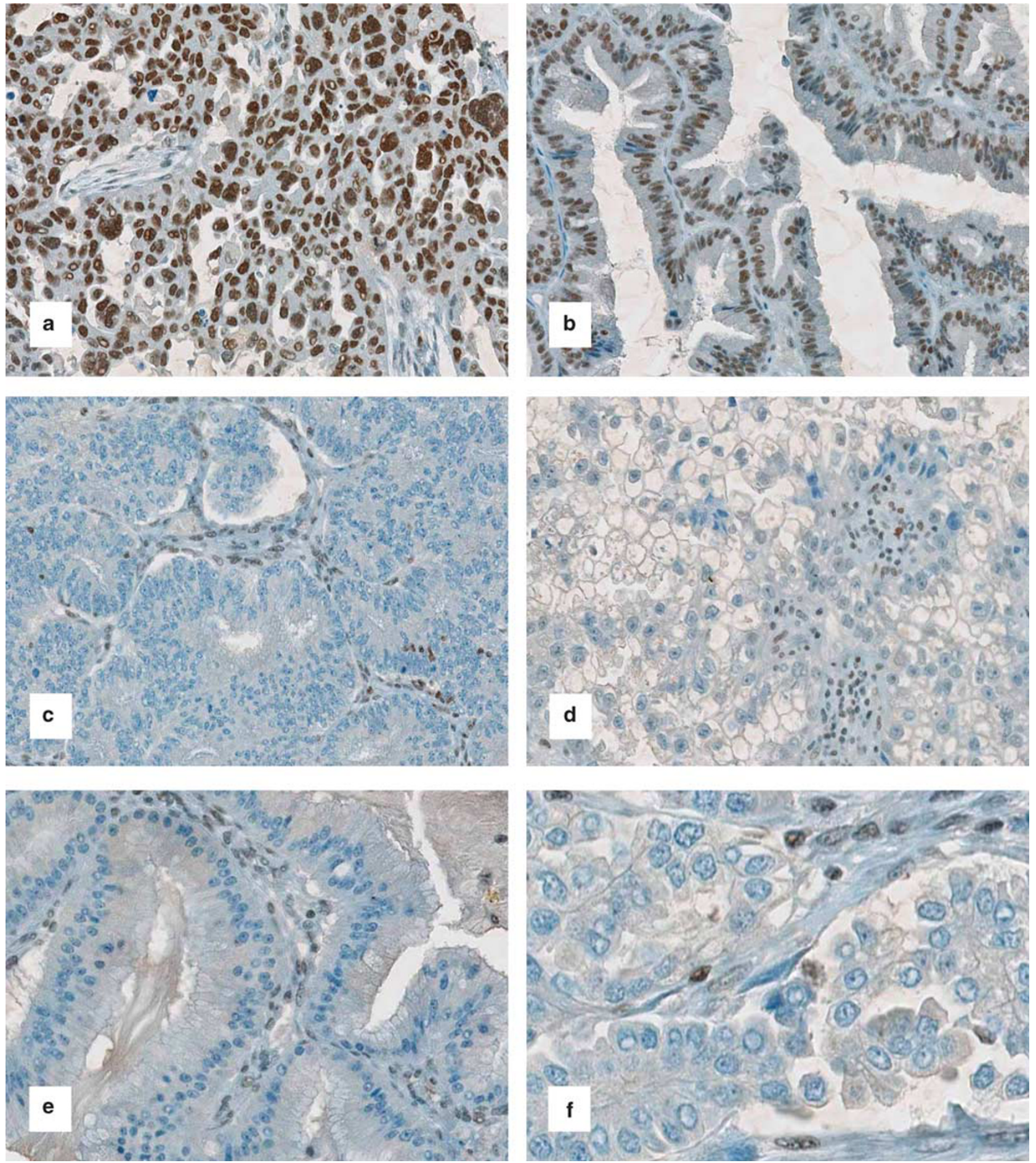

Figure 3 BAF250a expression in ovarian carcinomas. Immunohistochemical staining of ARID1A/BAF250a in ovarian carcinomas. (a) Example of strong nuclear expression in a high-grade serous carcinoma $(\times 200)$. (b) Moderate intense expression in a mucinous carcinoma $(\times 200)$. $(\mathbf{c}-\mathbf{e})$ Loss of expression in an endometrioid carcinoma $(\mathbf{c}, \times 200)$, in a clear cell carcinoma $(\mathbf{d}, \times 200)$, in a mucinous carcinoma $(\mathbf{e}, \times 200)$ and a serous carcinoma $(\mathbf{f}, \times 200)$. Note that only stromal cells show immunoreactivity.

using Sanger sequencing. We found complete lacking of BAF250a expression in three of the examined endometriomas and in one deep-infiltrating endometriotic lesion. Moreover, we observed that in most of the partly negative BAF250a cases lack of expression occurred in the form of grouped cell clusters.
According to Guan et al, ${ }^{11}$ this cluster-like negative expression pattern indicates a clonal loss of BAF250a in the concerned cells and happens only in ARID1A mutated clones.

Recent studies support that serous ovarian tumors arise from implantation of epithelium (benign or 
Table 2 Association of BAF250a (ARID1A) expression with clinico-pathological features in ovarian carcinomas

\begin{tabular}{|c|c|c|c|c|}
\hline \multirow[t]{2}{*}{ Characteristics } & \multirow[t]{2}{*}{$\begin{array}{l}\text { All cases } \\
\mathrm{n}(\%)\end{array}$} & \multicolumn{2}{|c|}{$\begin{array}{c}\text { BAF250a } \\
\text { expression } \mathrm{n}(\%)\end{array}$} & \multirow[t]{2}{*}{ P-value } \\
\hline & & Negative & Positive & \\
\hline All carcinomas & $129(100 \%)$ & $29(23)$ & $100(78)$ & \\
\hline $\begin{array}{l}\text { Age at diagnosis } \\
\quad \leqslant 60 \text { years } \\
\quad>60 \text { years }\end{array}$ & $\begin{array}{l}63 \\
66\end{array}$ & $\begin{array}{r}21(33) \\
8(12)\end{array}$ & $\begin{array}{l}42(67) \\
58(88)\end{array}$ & 0.004 \\
\hline $\begin{array}{l}\text { Histological type } \\
\text { Clear cell } \\
\text { Endometrioid } \\
\text { Serous } \\
\text { Mucinous }\end{array}$ & $\begin{array}{l}23 \\
28 \\
63 \\
15\end{array}$ & $\begin{aligned} 5 & (22) \\
13 & (46) \\
7 & (11) \\
4 & (27)\end{aligned}$ & $\begin{array}{l}18(78) \\
15(54) \\
56(89) \\
11(73)\end{array}$ & 0.003 \\
\hline $\begin{array}{l}\text { Tumor stage }(\mathrm{n}= \\
\text { PT1 } \\
\text { PT2 } \\
\text { PT3 }\end{array}$ & $\begin{array}{l}\text { 28) } \\
31 \\
15 \\
82\end{array}$ & $\begin{array}{r}10(32) \\
7(47) \\
12(15)\end{array}$ & $\begin{array}{r}21(68) \\
8(53) \\
70(85)\end{array}$ & 0.008 \\
\hline $\begin{array}{l}\text { Nodal stage }(\mathrm{n}= \\
\quad 0 \\
1\end{array}$ & 4) $\begin{array}{l}60 \\
34\end{array}$ & $\begin{array}{r}18(30) \\
4(12)\end{array}$ & $\begin{array}{l}42(70) \\
30(88)\end{array}$ & 0.045 \\
\hline $\begin{array}{l}\text { FIGO stage }(\mathrm{n}=1 \\
\quad 1 \\
2 \\
3 \\
4\end{array}$ & $\begin{array}{r}30 \\
9 \\
46 \\
38\end{array}$ & $\begin{array}{r}11(37) \\
5(56) \\
8(17) \\
4(11)\end{array}$ & $\begin{array}{r}19(63) \\
4(44) \\
38(83) \\
34(90)\end{array}$ & 0.005 \\
\hline $\begin{array}{l}\text { Tumor grade } \\
\quad 1 \\
2 \\
3\end{array}$ & $\begin{array}{l}23 \\
51 \\
55\end{array}$ & $\begin{array}{r}8(28) \\
12(24) \\
9(16)\end{array}$ & $\begin{array}{l}15(65) \\
39(77) \\
46(84)\end{array}$ & 0.201 \\
\hline $\begin{array}{l}\text { Residual disease } \\
\quad \text { R0 } \\
\text { R1 } \\
\text { R2 }\end{array}$ & $\begin{array}{c}n=87) \\
25 \\
27 \\
34\end{array}$ & $\begin{array}{r}10(40) \\
6(22) \\
2(6)\end{array}$ & $\begin{array}{l}15(60) \\
21(78) \\
32(94)\end{array}$ & 0.006 \\
\hline
\end{tabular}

${ }^{\mathrm{a}} \chi^{2}$ test.

malignant) from the fallopian tube. ${ }^{6}$ This theory also supports the fact that ovarian clear cell carcinomas and endometrioid ovarian carcinomas represent a different entity and that ovarian endometriotic inclusion cysts (endometriomas) are regarded as precursor of these tumors, originating from retrograde menstruation. ${ }^{6}$ There is evidence that eutopic endometrium in women with endometriosis exhibits intrinsic molecular abnormalities such as activation of oncogenic pathways. ${ }^{1,6}$ Some epidemiological studies demonstrated a lower risk for ovarian clear cell carcinomas and endometrioid ovarian carcinomas after tubal ligation or hysterectomy. ${ }^{30-32}$ A recent proposition for an ovarian cancer risk-assessment tool integrated endometriosis as well as no tubal ligation each as one of totally eight conditions associated with ovarian cancer. ${ }^{33}$

ARID1A mutations are supposed to have a key role in the formation of endometriosis-associated ovarian carcinomas and are probably occurring early, already at the stage of endometriosis. ${ }^{7}$ Ovarian cancer arises from the inclusion of epithelial cells into the ovarian stromal tissue. ${ }^{6}$ It seems evident that cluster-like ARID1A mutated clones in such inclusion cysts could therefore have a key role in the malignant transformation of these cysts. ${ }^{6,7,11}$ We demonstrate that complete loss of BAF250a expression occurs in a minority of endometriosis cases, mainly in endometriomas, without any evidence of malignant diagnosis at the moment of the operation. Additionally, we demonstrate that cluster-like loss of BAF250a expression can be shown in some further cases, implicating a clonal BAF250a expression deficiency. ${ }^{11}$ By these results, we corroborate that BAF250a expression deficiency in endometriosis is not restricted to tumor contiguous lesions and that loss of BAF250a expression can be observed already in benign endometriotic lesions, and could therefore indicate a risk of malignant transformation.

However, many questions need still to be answered, principally about the clinical consequences and the reasonability of a BAF250a screening in (ovarian) endometriosis. Further prospective and larger clinical studies are needed to answer these questions.

\section{Disclosure/conflict of interest}

The authors declare no conflict of interest.

\section{Acknowledgements}

This study was supported by Grants from the EMDOand Hartmann-Mueller-Foundations. We thank Silvia Behnke and Martina Storz for their excellent technical assistance. We also thank Prof B Seifert, PhD, Institute for Biostatistics University of Zurich, Switzerland, for the statistical support in this study.

\section{References}

1 Bulun SE. Endometriosis. N Engl J Med 2009;360: 268-279.

2 Giudice LC, Kao LC. Endometriosis. Lancet 2004;364: 1789-1799.

3 Anglesio MS, Carey MS, Köbel M, et al. Clear cell carcinoma of the ovary: a report from the first Ovarian Clear Cell Symposium, June 24th, 2010. Gynecol Oncol 2011;121:407-415.

4 Kobayashi H, Sumimoto K, Moniwa N, et al. Risk of developing ovarian cancer among women with ovarian endometrioma: a cohort study in Shizuoka, Japan. Int J Gynecol Cancer 2007;17:37-43.

5 Ness RB. Endometriosis and ovarian cancer: thoughts on shared pathophysiology. Am J Obstet Gynecol 2003;189:280-294.

6 Kurman RJ, Shih IM. The origin and pathogenesis of epithelial ovarian cancer: a proposed unifying theory. Am J Surg Pathol 2010;34:433-443. 
7 Wiegand KC, Shah SP, Al-Agha OM, et al. ARID1A mutations in endometriosis-associated ovarian carcinomas. N Engl J Med 2010;363:1532-1543.

8 Jones S, Wang TL, Shih IM, et al. Frequent mutations of chromatin remodeling gene ARID1A in ovarian clear cell carcinoma. Science 2010;330:228-231.

9 Wang X, Nagl Jr NG, Flowers S, et al. Expression of p270 (ARID1A), a component of human SWI/SNF complexes, in human tumors. Int J Cancer 2004; 112:636.

10 Reisman D, Glaros S, Thompson EA. The SWI/SNF complex and cancer. Oncogene 2009;28:1653-1668.

11 Guan B, Mao TL, Panuganti PK, et al. Mutation and loss of expression of ARID1A in uterine low-grade endometrioid carcinoma. Am J Surg Pathol 2011;35: 625-632.

12 Kononen J, Bubendorf L, Kallioniemi A, et al. Tissue microarrays for high-throughput molecular profiling of tumor specimens. Nat Med 1998;4:844-847.

13 Maeda D, Mao T-L, Fukayama M, et al. Clinicopathological significance of loss of ARID1A immunoreactivity in ovarian clear cell carcinoma. Int J Mol Sci 2010;11:5120-5128.

14 Weichert W, Roske A, Gekeler V, et al. Association of patterns of class I histone deacetylase expression with patient prognosis in gastric cancer: a retrospective analysis. Lancet Oncol 2008;9:139-148.

15 Somigliana E, Vigano' P, Parazzini F, et al. Association between endometriosis and cancer: a comprehensive review and a critical analysis of clinical and epidemiological evidence. Gynecol Oncol 2006;101: 331-341.

16 Vlahos NF, Kalampokas T, Fotiou S. Endometriosis and ovarian cancer: a review. Gynecol Endocrinol 2010;26:213-219.

17 Sampson JA. Endometrial carcinoma of the ovary, arising in endometrial tissue in that organ. Arch Surg 1925;10:1-72.

18 Brinton LA, Gridley G, Persson I, et al. Cancer risk after a hospital discharge diagnosis of endometriosis. Am J Obstet Gynecol 1997;176:572-579.

19 Melin A, Sparén P, Persson I, Bergqvist A. Endometriosis and the risk of cancer with special emphasis on ovarian cancer. Hum Reprod 2006;21:1237-1242.

20 Vercellini P, Parazzini F, Bolis G, et al. Endometriosis and ovarian cancer. Am J Obstet Gynecol 1993;169: 181-182.
21 Ogawa S, Kaku T, Amada S, et al. Ovarian endometriosis associated with ovarian carcinoma: a clinicopathological and immunohistochemical study. Gynecol Oncol 2000;77:298-304.

22 Kumar S, Munkarah A, Arabi H, et al. Prognostic analysis of ovarian cancer associated with endometriosis. Am J Obstet Gynecol 2011;204:63e1-63e7.

23 Lim MC, Chun KC, Shin SJ, et al. Clinical presentation of endometrioid epithelial ovarian cancer with concurrent endometriosis: a multicenter retrospective study. Cancer Epidemiol Biomarkers Prev 2010;19: 398-404.

24 Aris A. Endometriosis-associated ovarian cancer: a ten-year cohort study of women living in the Estrie Region of Quebec, Canada. J Ovarian Res 2010;3:2.

25 Köbel M, Kalloger SE, Huntsman DG, et al. Differences in tumor type in low-stage versus high-stage ovarian carcinomas. Int J Gynecol Pathol 2010;29:203-211.

26 Itamochi H, Kigawa J, Terakawa N. Mechanisms of chemoresistance and poor prognosis in ovarian clear cell carcinoma. Cancer Sci 2008;99:653-658.

27 Jacoby VL, Fujimoto VY, Giudice LC, et al. Racial and ethnic disparities in benign gynecologic conditions and associated surgeries. Am J Obstet Gynecol 2010; 202:514-521.

28 Sugiyama T, Kamura T, Kigawa J, et al. Clinical characteristics of clear cell carcinoma of the ovary: a distinct histologic type with poor prognosis and resistance to platinum-based chemotherapy. Cancer 2000;88:2584-2589.

29 Birrer MJ. The origin of ovarian cancer-is it getting clearer? N Engl J Med 2010;363:1574-1575.

30 Rosenblatt KA, Thomas DB. Reduced risk of ovarian cancer in women with a tubal ligation or hysterectomy. The World Health Organization Collaborative Study of Neoplasia and Steroid Contraceptives. Cancer Epidemiol Biomarkers Prev 1996;5:933-935.

31 Miracle-McMahill HL, Calle EE, Kosinski AS, et al. Tubal ligation and fatal ovarian cancer in a large prospective cohort study. Am J Epidemiol 1997;145:349-357.

32 Hankinson SE, Hunter DJ, Colditz GA, et al. Tubal ligation, hysterectomy, and risk of ovarian cancer. A prospective study. JAMA 1993;270:2813-2818.

33 Vitonis AF, Titus-Ernstoff L, Cramer DW. Assessing ovarian cancer risk when considering elective oophorectomy at the time of hysterectomy. Obstet Gynecol 2011;117:1042-1050. 